\title{
Reconfigurable Wireless Sensor Node with Improved Autonomous Linearization
}

\author{
Anuradha C. Ranasinghe, Lahiru K. Rasnayake, M. Kalyanapala
}

\begin{abstract}
The usage of reconfigurable hardware in wireless sensor networks plays an important role which enables multi parameter estimation in complex environments. The need for vast sensor compatibility and sensor fusion will become crucial requirements as wireless sensor networks become autonomous and intelligent. Due to the non linear behaviour of sensors and analogue circuitry in the front end, such applications often require a rigorous software model to perform sensor linearization for best accuracy. This paper presents an implementation of a reconfigurable wireless sensor node based on a CMOS analogue multiplexer network supporting universal functions for different industrial sensors while being compact and energy efficient, which is conducive to wireless sensor networking. Further an accurate optimized and autonomous linearization technique based on rational interpolation is also presented in order to compensate non idealities of the sensor node. The concept, simulation results, prototype implementation with industrial components and the results of system integration are discussed in this paper to illustrate potential applications in mass scale data acquisition based on wireless sensor networks.
\end{abstract}

Keywords - WSN, Non Linear Sensors, Reconfigurable Hardware, Autonomous Systems, Rational Interpolation, Multiplexers

\section{INTRODUCTION}

In the modern world, the combination of multi functioning data acquisition systems and wireless communication protocols provide creative means to develop energy efficient, intelligent wireless sensor nodes for industrial applications. The ability to interact with the physical environment in wireless domain provides more robust and versatile platform over traditional environmental monitoring scheme. Reconfigurable hardware technologies have been recently introduced to wireless sensor networks (WSN) for robust, low power operation while enabling multi parameter data acquisition for a given node. A single sensor node may be designed integrating a sensor element, signal conditioner and Network Application Processor (NCAP) into one sensor node. Single sensor MEMS (Micro-electro-mechanical Sensor) chips and motes are some examples for such a system [1]. If a node per sensor approach is used, many nodes are required in case different parameters of a certain area are to be monitored. Maintaining such a WSN is quite expensive as well as increases the complexity of the network. The traditional approach to provide multi sensor capability for a WSN node is by employing several signal conditioning stages to the front end of the circuit where every sensor interface is

Manuscript received February, 16, 2014. Recommended by Dr. Nalin Ranasinghe on July 28, 2014.

Anuradha C. Ranasinghe, Lahiru K. Rasnayake, M. Kalyanapala are with Department of Electrical and Computer Engineering, Sri Lanka Institute of Information Technology, New Kandy Road, Malabe, Sri Lanka (e-mails: anuradha.r@sliit.lk, lahiru.r@sliit.lk, kalyanapala.m@sliit.lk) dedicated to a certain sensor type. Compared to traditional hardware design topologies, reconfigurable hardware designs have shown a certain improvement of designing more flexible devices [2]. Field programmable gate array (FPGA) based designs are more popular for digital sensors, though they require additional components to facilitate multi sensing operation in mixed signal domain [3]. In the analog domain, two major programmable hardware technologies are the field programmable analog arrays (FPAA) and programmable mixed signal system on chip technology (e.g. PSoC). Both technologies are capable of providing all the common components for supporting different mixed signal processing applications [4].

\section{RELATED WORK}

The development of an autonomous wireless sensor node involves the embedding self-adjustment functionalities that should be able to fix non idealities of the system such as offset, variations in gain and non linearity for rigorous operation. Such a system should utilize the least amount of time and power for readjusting process. An accepted practice utilized in the past by measurement system designers was to linearize the sensor signal in order to compensate for system non idealities. The subject of linearization of measurement systems has been considered on different forms and stages, basically in the design of circuits with MOS and CMOS technologies [5-6]. Studied cases included the usage of auxiliary hardware and programmable software solutions to evaluate the linearization performance of measurement systems. One application suggests analogue to digital converters to solve nonlinearities at the same time the conversion is made [7-8]. Further, ROM memories have been used to save data tables and optimize lookup tables to solve linearization problems [9-10]. Simple resistor divider technique and a programmable hardware linearization method were also presented [11-12]. The self-calibration concept using artificial neural networks is approached from different perspectives: simulation of auto calibration results [13] and works related with auto calibration of specific sensors [14-15]. Some of these cannot be easily implemented on a low power microcontroller $(\mu \mathrm{C})$. Several important works related to recursive algorithms that can be applied to the self readjustment of intelligent sensors exist based on different type interpolation techniques. Among the simplest algorithms, the three point calibration method [16-17] suggests a simple point by point calibration technique for single and multidimensional data set. Also the progressive polynomial calibration method [18] and its improved version [19] were proven to be efficient methods in terms of computation burden and optimized accuracy. However achieving a highly non linear transfer function becomes difficult due to the large set of calibration points required at initial stage. 
Recent studies [20] show that the use of rational polynomial interpolation for transfer function modelling has a better approximation for a given data set with the minimum least square error compared to polynomial approximations methods. Compared with other techniques such as Lagrangian Interpolation or Gauss Newton Approximation, the asymptotic behaviour and better interpolation ability of rational functions makes it ideal for modelling highly non linear characteristics of systems where the transfer function of the system can be expressed with least number of terms.

To counteract previously mentioned issues, we propose a design topology of a reconfigurable wireless sensor node which was designed based on ultra low power analogue components. The software of the central processing unit incorporates point by point linearization method which autonomously determines the best transfer curve for sensors by given calibration points. By using programmable mixed signal hardware architecture, this new approach enables a single channel of this node to obtain signals from a vast diversity of sensors. The most significant advantage would be the autonomous transfer function identification of non linear sensors. It also ensures homogeneity among WSN nodes.

\section{CONCEPT OF RECONFIGURABLE INTERFACE}

In the proposed system, the flexibility of reconfigurable interface is essentially important since it decides the universal functionality and application scope of the data acquisition process of WSN node. The interface circuit was designed to accommodate various types of sensor output signals, provide sensor driving, offset adjustment, data linearization and automatic gain to match the signals and sensitivities of different sensor elements. The trade-off between the measurement accuracy and the flexibility should be optimized so that the resulting system can be widely adaptable to the multi parameter system. Moreover power management of the device is critically important since WSN nodes are deployed in an energy constrained environment [21]. Fig. 1 shows the hardware block diagram of the WSN node where dedicated signal conditioning stages in traditional design were replaced by universal transducer interface module (UTIM).

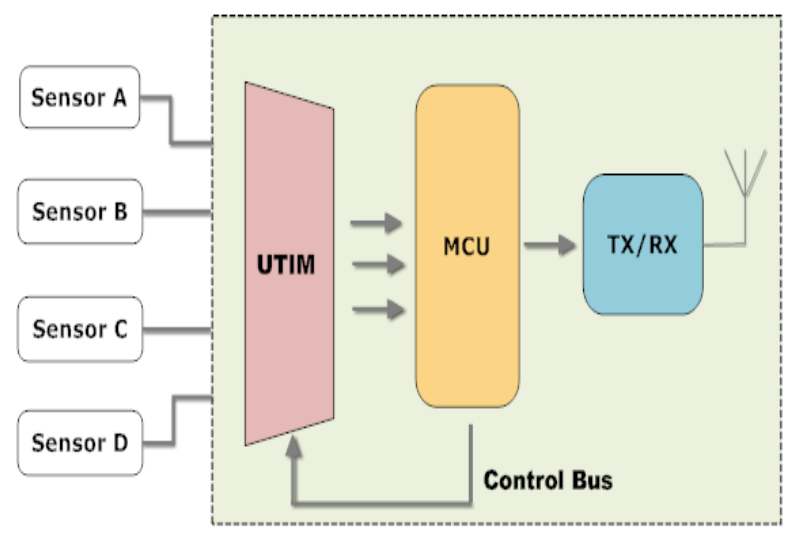

Fig. 1 Proposed WSN Node Design

As seen from Fig. 1, the most distinct advantage of this design is that this can be further miniaturized through an integrated mixed circuit design, even though a modular approach was employed in the current prototype.

\section{A. Mixed Signal Circuit Design}

The UTIM is based on an analog multiplexer network which facilitates the required functionality to perform signal routing between sensor signals and amplifier stages. Time-divisionmultiplexing will be used to take analog sensor signals from a distributed sensor array periodically so that data can be processed on a common transmission line in a digitally encoded format. These multiplexers were chosen from CMOS logic family because of its high noise immunity and ultra low power profile, where the design can be fabricated to be compact with low power, cost effective implementation. Also UTIM uses the architecture to synchronize the multiplexing and sampling to ensure a proper settling of the analog data signals while maintaining energy efficiency.

The use of dynamic sensor power cycling with UTIM provides an opening for reducing average power consumption in applications where energy use must be tightly managed and power hungry sensors are interfaced. The average power dissipation, of a transducer is quantified by the following equation:

$$
P_{A V G}=D P_{O N}+(1-D) P_{O F F}
$$

where $D, P_{O N}$, and $P_{O F F}$ represent duty cycle, power in normal operation and power in off-mode of the transducer respectively. Selecting appropriate duty cycle by considering settling time of the sensor, the average power of the entire system can be managed to operate in very low level. For instance, considering a load cell (VISHAY Model 9010), $P_{A V G}$ can be greatly minimized while maintaining the measurement accuracy. Table 1 shows the technical data of the load cell.

TABLE 1

TECHNICAL DATA OF THE LOAD CELL (VISHAY MODEL 9010)

\begin{tabular}{|l|l|}
\hline Parameter & \multicolumn{1}{|c|}{ Value } \\
\hline Settling Time & $150 \mathrm{mS}$ (Typical) \\
\hline Operating Power & $240 \mathrm{~mW}$ \\
\hline Off-Power & $6.8 \mu \mathrm{W}$ \\
\hline
\end{tabular}

Taking $T_{\text {on }}$ (Power-on Time) $=160 \mathrm{mS}$ (settling time $+10 \mathrm{mS}$ acquisition time), and $T_{\text {on+off }}($ Cycle time) $=1 S$ the average power can be written:

$$
\begin{aligned}
P_{A V G} & =(0.16 \times 240)+(1-0.16) 0.0068 m W \\
P_{A V G} & =38.405 m W
\end{aligned}
$$

The average power consumed by the sensor is approximately $16 \%$ of its normal operation power. Hence dynamic power cycling is proven to be energy efficient with the UTIM circuit. The initial design shown in fig. 2 is configured for four analogue channels. Each analogue channel consist of 4-wire interface, where Ex+, Ex-, Sen+ and Sen-correspond to the terminals of excitation supplies and differential sensor signal respectively. These differential signals will be converted into a single ended voltage by routing the signal to an appropriate analog block. Each analog block was design for a specific sensor type which may include Op-Amps, discrete component to provide signal conditioning and filtering before fed into the ADC of the processing unit (MCU). The basic UTIM design may require an optional multiplexer stage, so that only one ADC channel is sufficient to digitize the sensor analog signal. 


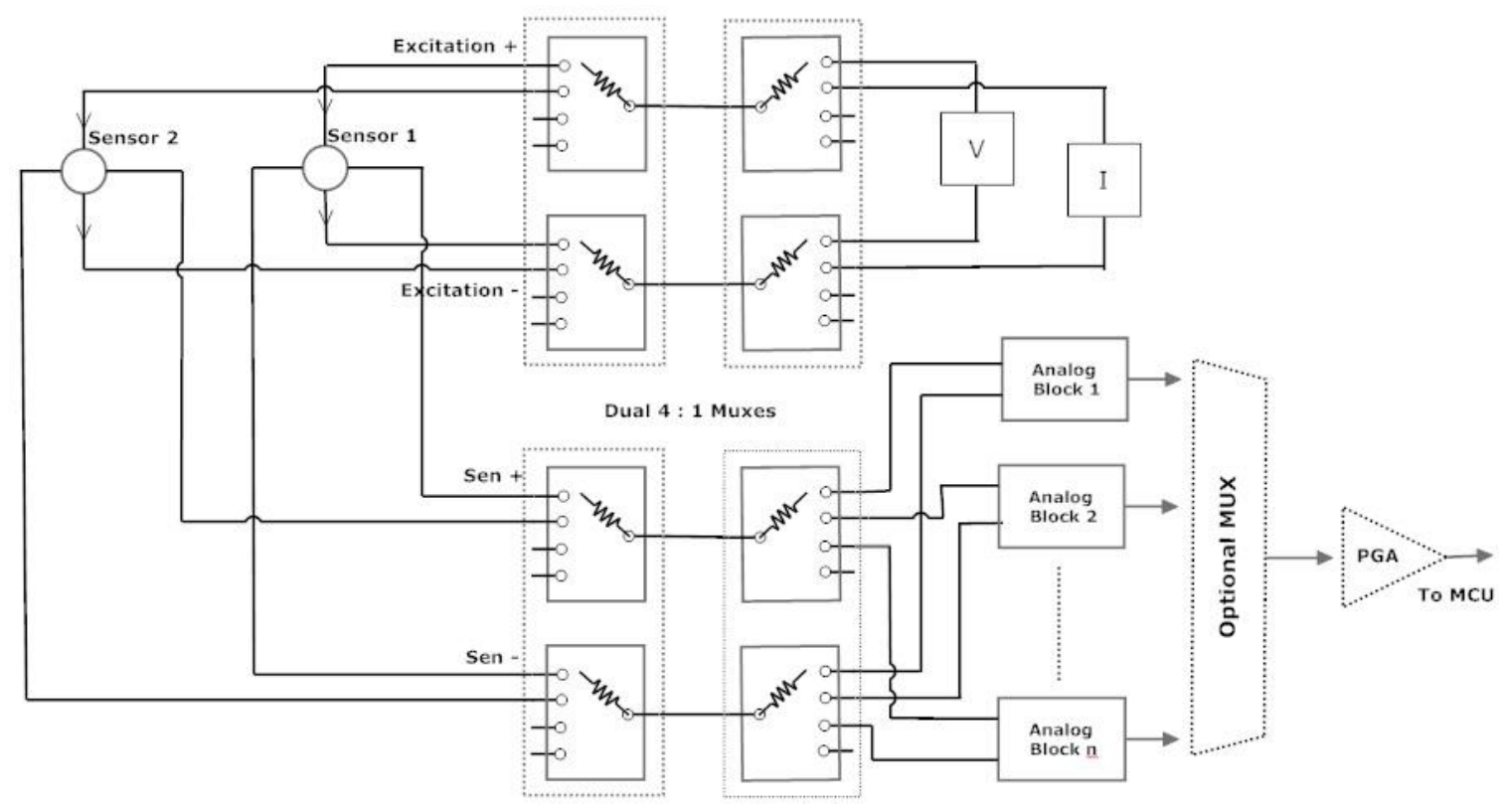

Fig. 2 UTIM Based on Analogue Components at Front End

\section{B. Signal Conditioning Blocks}

The multi functioning capability of UTIM is achieved by integrating different signal conditioning blocks at the back end of multiplexer network. These intermediate blocks were made of analog functioning integrated circuits such as operational amplifiers; each block converts the sensor signal into a voltage parameter, matches impedances and scales the signal level prior to the ADC input. These analog blocks were designed based on the operational principle and the type of central element of the sensor, so that each block provides more general functions rather than being a dedicated interface type. Besides that, two independent excitation supplies were provided, where source selection is optional. Figure 3 shows the UTIM operation for a typical bridge sensor.

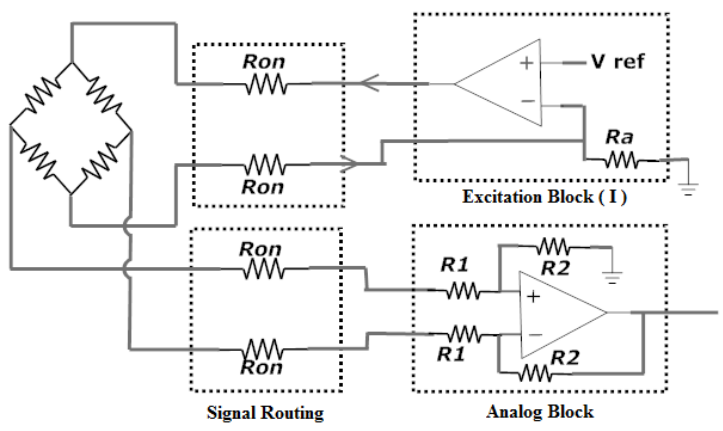

Fig. 3 Bridge Excitation (Constant Current)

Normally Bridge sensors generate a weak signal which represents change in resistance of the strain gauges. If the gauge is powered with voltage excitation, the voltage drops appear on Switch On resistance (Ron) of the multiplexer will cause a significant error to the measurement. In such a situation, it is adequate to drive the bridge with known current excitation where the Ron is not constant. Excitation Block (I) represents a basic current source device made with very few components. For single element varying bridge, the voltage output is given by;

$$
V_{O}=\frac{R_{2}}{R_{1}}\left[\frac{V_{R E F}}{R a} \Delta R\right]
$$

where $\Delta R$ is the resistance change of the bridge element due to the applied strain.

Programmable Gain Amplifier (PGA) is another important part of a data acquisition system. When dealing with wide range of signal levels, different gain ratios may be required to scale signals for better accuracy. Since most of PGAs available in the market are very expensive, we are proposing a low cost solution based on simple analog components. Figure 4 shows the design of PGA using a single op amp, analog mux with couple of discrete components for three different gain values.

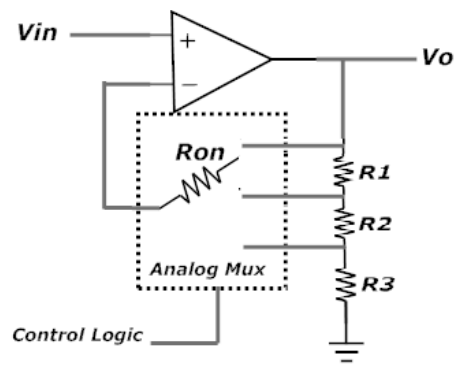

Fig. 4 Low Cost Programmable Gain Amplifier

The control bus line of the multiplexer should be maintained in a logic level to provide the required gain. This design has the advantage of bypassing Ron from the gain stage so that the variations of Ron (caused by thermal drifts, multiplexer input voltage levels) will not affect on the measurement. Better performance can be obtained using precision resistors for R1 to R3. System parameters relevant to this design such as frequency response and gain errors will be discussed in the device implementation stage.

\section{AUTONOMOUS LINEARIZATION TECHNIQUE}

Some sensors have the advantage of a very high sensitivity to changes in physical phenomena, and the disadvantage of an aggressively nonlinear characteristic. On the other hand this might introduce a non linear error to the measurement, which 
should be eliminated using a proper hardware design or software calibration. Some techniques based on numerical approximation methods have been discussed in early literature [22-28], include Lagrangian Interpolation Polynomials, Cubic Splines, Gauss Newton algorithm, Steepest Descent techniques. Generally for power constraint reconfigurable embedded systems, the least amount of time and computational burden always preferred in applying these algorithms.

Very little literature has been written with regard to rational functions so this area is mostly open for exploration to researchers and mathematicians. Rational approximation was found to be a successive method for linearizing highly non linear data due to its asymptotic nature. The method presented here is a simplified version of the Thiele's continuous fraction algorithm which ultimately derives the transfer function of a sensor using conventional recursive divided differences for given dataset. The algorithm finds appropriate rational function after each calibration point utilizing a finite iterative technique. Because of this simple point by point interpolation process, the algorithm does not require memory to hold large matrices and computational loads such as matrix inversion etc.

In the first step, we would consider calibrating univariate response. The algorithm starts with taking divided difference between two calibration data points. Using triangle rule, we can form the divided difference coefficients according to format as in Table II given below.

Where;

TABLE II

CALIBRATION DATA SET

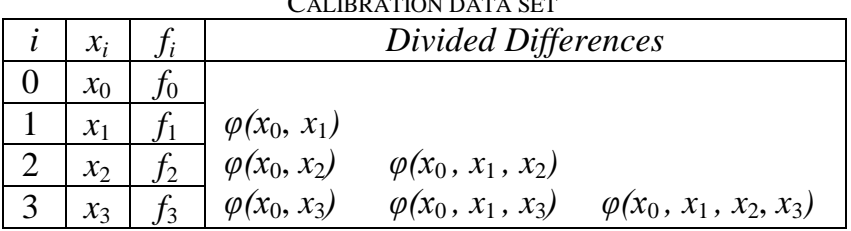

$f_{i}$ and $x_{i}$ represent dependent and independent variables of the sensor response

$$
\begin{gathered}
\varphi\left(x_{m}, x_{n}\right)=\frac{x_{n}-x_{m}}{f_{n}-f_{m}} \\
\varphi\left(x_{m} \ldots, x_{p}, x_{q}\right)=\frac{x_{q}-x_{p}}{\varphi\left(x_{m}, \ldots x_{o}, x_{q}\right)-\varphi\left(x_{m}, \ldots x_{o}, x_{p}\right)}
\end{gathered}
$$

Corresponding rational expression for each point can be recursively found according to the following procedure:

$$
\begin{aligned}
R_{n}(x)= & f_{0}+\frac{b_{1} \mid}{\mid a_{1}}+\frac{b_{2} \mid}{\mid a_{2}}+\ldots . .+\frac{b_{n} \mid}{\mid a_{n}} \\
& \left\{\begin{array}{l}
a_{0}=f_{0} \\
a_{n}=\varphi\left(x_{0}, \ldots, x_{n-1}, x_{n}\right) \\
b_{n}=\left(x-x_{n-1}\right)
\end{array}\right.
\end{aligned}
$$

where $a_{n}$ and $b_{n}$ are given by the coefficients of diagonal and numerator fractions respectively.

$$
\begin{gathered}
\left\{\begin{array}{l}
P_{-2}=0, P_{-1}=1, P_{0}=a_{0} \\
Q_{-2}=1, Q_{-1}=0, Q_{0}=1
\end{array}\right. \\
R_{j}=\frac{P_{j}}{Q_{j}}=\frac{a_{j} P_{j-1}+b_{j} P_{j-2}}{a_{j} Q_{j-1}+b_{j} Q_{j-2}}
\end{gathered}
$$

where $j=1 \ldots \ldots n-1, n=$ number of calibration points

Defining the continuous fraction given in (3) can be simplified to equation (4) which enables finding the final rational function for the entire data set.

When using the proposed algorithm, it is necessary to define its performance to satisfy certain criteria such as, number of calibration points, minimum non linearity error, and computational speed. In order to demonstrate the performance of the proposed method, a non linear data set was generated using an increasing exponential function to interpolate by the Simplified Rational Interpolation (SRI) algorithm in the first step. The evaluation has been carried out by changing the linearity of the function keeping number of calibration points constant. The result shown in figure 5 was obtained using 4 calibration points which clearly explains the behaviour of the non linear response.
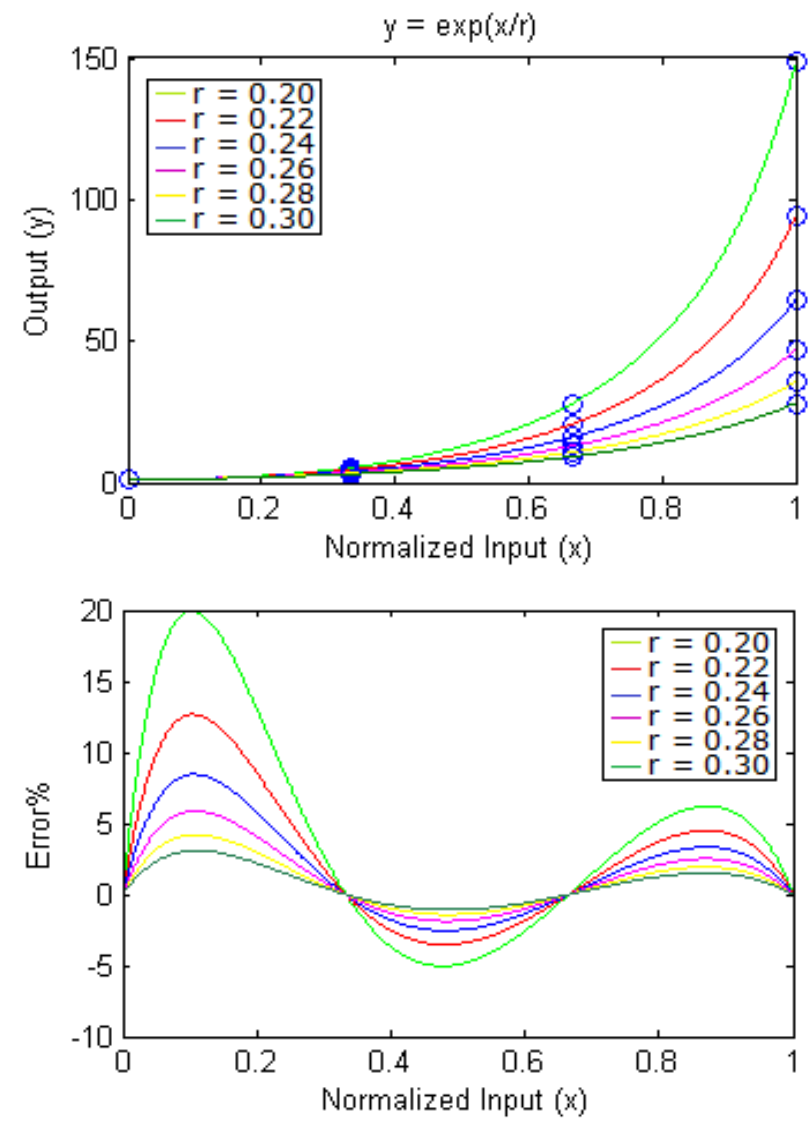

Fig. 5. The interpolates data and its error plot with proposed algorithm for a non linear function

Figure 5 clearly shows the interpolated result of the non linear data set and its corresponding error curves. Point A, B, C and $\mathrm{D}$ represent successive calibration points which have been used to determine the approximated rational function. Variable $r$ determines the non linearity of the function. Changing the non linearity by setting $r=0.2,0.4$ and 0.6 , the 
simulated error plot gives a good evaluation of the effectiveness of the algorithm. According to the error plots given in Fig 1, it can be noticed that $20 \%$ of maximum error has been reduced to $1 \%$ when the non linearity factor decreases by 0.2 .

Apart from that, linearization was attempted with popular Gauss Newton Algorithm (GNA), Polynomial Least Square Method (PLSM) [29-33] and Progressive Polynomial Method (PPM). To obtain an acceptable error for GN and PLSM method, a large number of data set, iterations and initial values were required. Compared to these methods, PPM which is a point by point calibration technique shows an acceptable accuracy of $75 \%, 4$ data points. When the non linearity is further reduced $(\mathrm{r}=0.4,0.6)$ the observed accuracies were $80 \%$ and $87 \%$ respectively. This shows that the SRI algorithm provides the best accuracy with the least required number of calibration data points and computational burden. Increasing the number of calibration points to six, we can see the nonlinear error of the generated data set can be greatly reduced as shown in Fig 6.

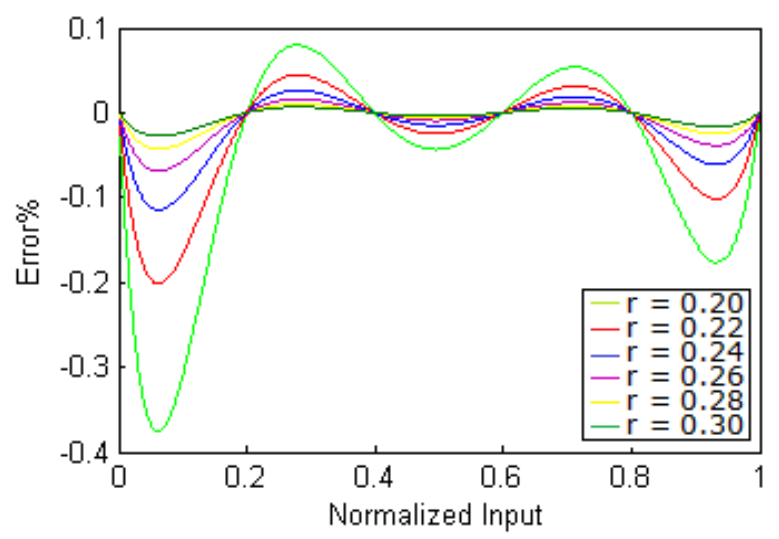

Fig. 6.Minimized error result with six calibration points.

With $r$ set to 0.2 , the maximum error of the data set was minimized to $0.37 \%$ which is a great benefit of the proposed algorithm. The result proves that even the highest non linearity can be greatly reduced by increasing number of points in the algorithm.

In the second step of the evaluation, the algorithm was applied to linearize a standard $3.3 \mathrm{k}$ thermistor using 4 calibration points. A thermistor is a good example for a non linear sensor which has considerable non linearity where the change in the measurement is most rapid at low temperatures, giving great resolution for determining the corresponding temperature values there. At the other end of the range, resistance levels change relatively less with temperature and measurement resolution is relatively poor. The sensor data set was taken from datasheet recommended by National Institute of Standard and Technology (NIST) [34]. Points were selected to cover its entire temperature range. The resistance value of the thermistor and corresponding temperature in Kelvin were selected as independent and dependent variables respectively. Following table shows the data set and their inverted differences. Four calibration points were selected from the resistance range representing temperature values ranging from $273 \mathrm{~K}$ to $360 \mathrm{~K}$.
TABLE III

THERMISTOR EXAMPLE

\begin{tabular}{|r|r|c|rrr|}
\hline$i$ & \multicolumn{1}{|c|}{$R(\Omega)$} & $T(K)$ & \multicolumn{3}{|c|}{ Divided Differences } \\
\hline 0 & 341 & 359.26 & & & \\
\cline { 1 - 3 } 1 & 3643 & 295.93 & -52.14 & & \\
\cline { 1 - 3 } 2 & 7126 & 281.48 & -87.23 & -99.25 & \\
\cline { 1 - 3 } 3 & 10481 & 273.71 & -118.53 & -103.00 & -849.09 \\
\hline
\end{tabular}

Using eq(3), (4) and (6) the rational function can be computed step by step according to the following format.

$$
\begin{gathered}
j=1 ; \quad \frac{P_{1}}{Q_{1}}=\frac{a_{1} P_{0}+b_{1} P_{-1}}{a_{1} Q_{0}+b_{1} Q_{-1}}=365.8-0.012 x \\
j=2 ; \quad \frac{P_{2}}{Q_{2}}=\frac{a_{2} P_{1}+b_{2} P_{0}}{a_{2} Q_{1}+b_{2} Q_{0}}=\frac{260.01 x-5.84 \times 10^{5}}{x+1531.81} \\
j=3 ; \quad \frac{P_{3}}{Q_{3}}=\frac{a_{3} P_{2}+b_{3} P_{1}}{a_{3} Q_{2}+b_{3} Q_{1}}= \\
\frac{-x^{2}+2.59 \times 10^{5} x-3.86 \times 10^{8}}{946.23 x+9.98 \times 10^{5}}
\end{gathered}
$$

The obtained rational function by the SRI algorithm and conventional Steinhart model for $3.3 \mathrm{k}$ thermistor were compared with sensor data to evaluate the performance of the proposed method. The result from the simulation is shown in Fig 7.
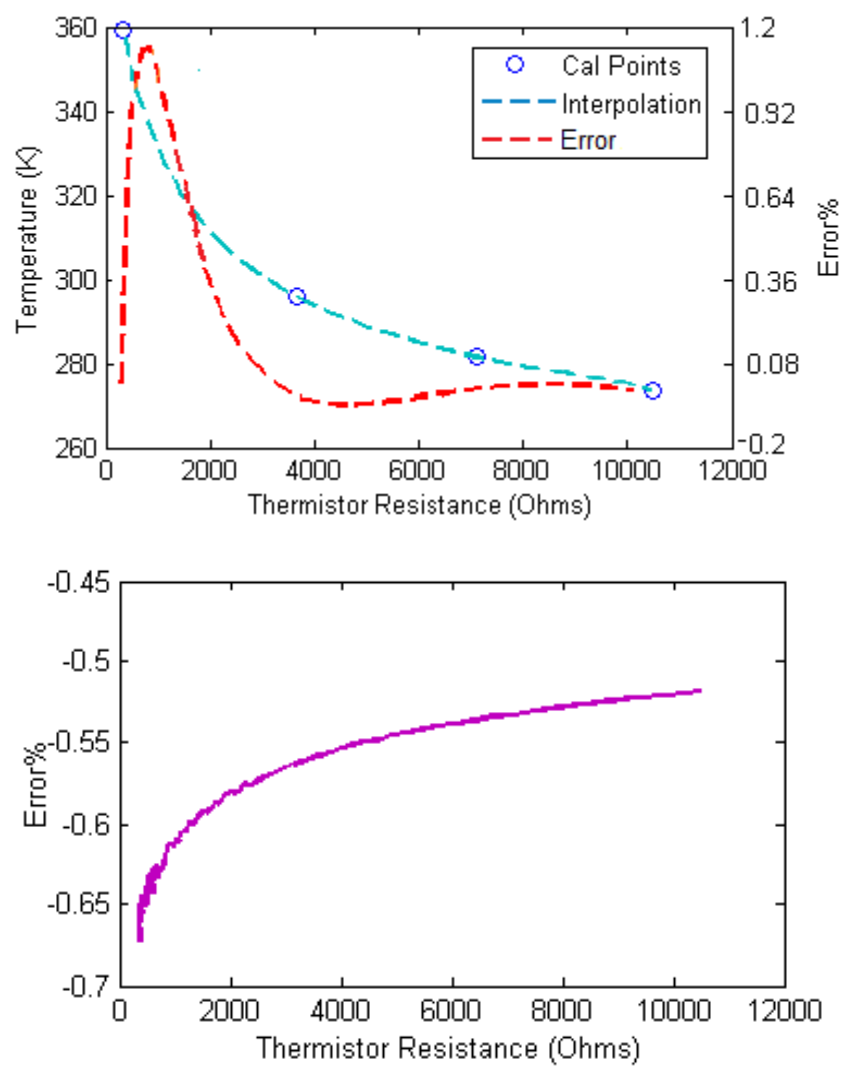

Fig. 7.Interpolated result compared with Stein Hart Equation

The error is significantly much smaller in terms of percentage when compared with other algorithms described previously and as seen in the figure, the SRI algorithm, with its lower 
computation time is a significant leap in deriving the sensor's characteristic function.

The simulation results show that approximately $98 \%$ accuracy (1.14\% of maximum error) can be achieved with 4 calibration points of the SRI algorithm. Compared to Steinhart model, SRI result gives a moderate value. If the computational workload is considered, the SRI algorithm has robust simplification steps rather than using logarithmic functions of Steinhart model for time constraint applications. The proposed algorithm was tested on an ARM Cortex M3 microprocessor setting its operating frequency to $10 \mathrm{MHz}$ which is ideal for low power operating WSN applications. To find coefficients (divided differences) at the initial stage, the microprocessor required $119 \mathrm{uS}$. Only $84 \mathrm{uS}$ execution time was required to compute the rational function at each recursion cycle. In contrast, Steinhart equation required 3.32 $\mathrm{mS}$ for its logarithmic function, which makes the new SRI algorithm efficient in saving computational burden. However it is also important to note the convergence of the proposed method with minimum number of calibration points in order to evaluate the efficiency. The same example for thermistor can be used for this. Next figure shows the simulation result of the maximum error with respect to the calibration points used to interpolate the data set.

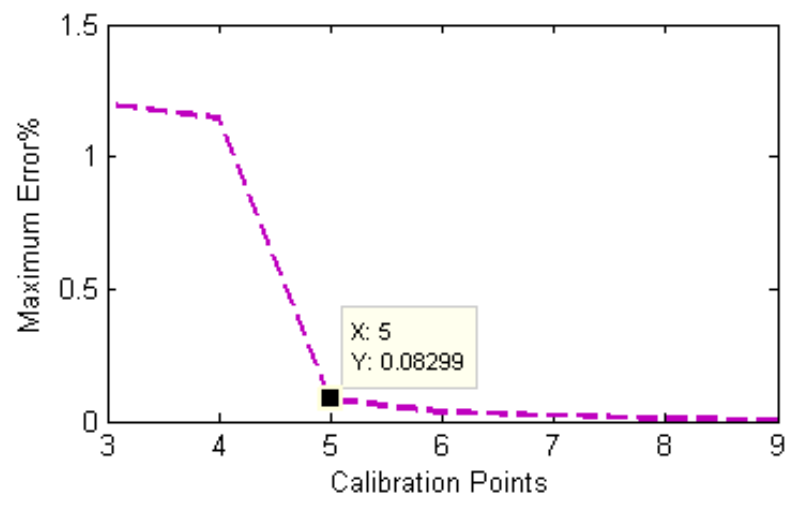

Fig. 8.Convergent Property.

Increasing the number of calibration points to 5, shows a dramatical change in maximum error of the interpolated data set. The maximum error of $1.14 \%$ for 4 point calibration has been minimized to $0.083 \%$ by increasing the number of points to 5, which is a great benefit of the proposed method.

\section{OVERALL PERFORMANCE \& PROTOTYPE DESIGN}

Since the emphasis of this paper is focused on suggesting an autonomous linearization technique for a reconfigurable WSN system, the user may select and integrate other auxiliary peripherals for the WSN node, depending on the application requirements and feasibility. In developing the experimental prototype for this research, the paramount concern was creating a low power design, with universal capability and optimized accuracy. The effectiveness of the algorithm will be proven later in terms of processing speed and convergence. The components used for the UTIM included Dual 4:1 CMOS Analog Multiplexers (MAX4618) for the signal routing stage and a micro power op-amp (LT1079) for optimum power efficiency in this prototype. The performance of this UTIM was tested with the LPC1769 ARM Cortex M3 micro processor from NXP and the XBee
ZB module. The LPC1769 has ultra low power profiles for different sleep modes. The power consumption of the UTIM with respect to its switching frequency with different sensor attachments was recorded as shown Fig 9.

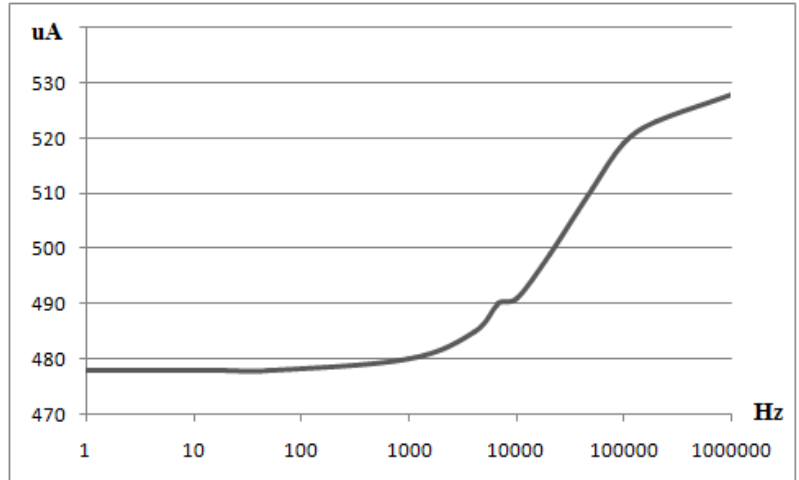

Fig. 9 Current Consumption of UTIM.

With the current consumption given in micro amperes (uA) and channel switching frequency in Hertz $(\mathrm{Hz})$ in log-scale, the observations prove that the UTIM is highly power efficient for typical industry grade applications since the current consumption can be maintained as low as $478 \mathrm{uA}$ for adequate switching frequencies. The device performance in high frequencies is determined by the frequency responses of individual components. To evaluate the frequency response of the measurement chain, we feed a sinusoidal signal to the input and record the signal output at final stage by setting PGA gain to 1, 10 and 100 respectively. The gain error of the final output with respect to the input signal frequency is shown in Fig 10.

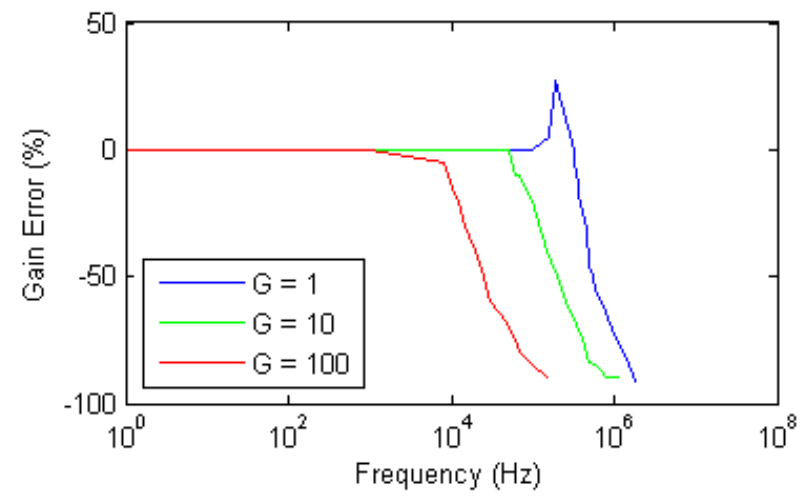

Fig. 10 Frequency Response of the Prototype Design.

As shown in Fig 10, when PGA gain is set to 1 (1\% tolerance resistors were used) the operational range of the device is limited to $200 \mathrm{kHz}$. Increasing gain to 10 and 100 subsequently reduces the operation range to $50 \mathrm{kz}$ and $8 \mathrm{kHz}$ respectively. This behaviour can be expected in micro power operational amplifiers since the power optimization of VLSI design lead to a reduction in gain bandwidth product of the component. However this frequency limit is more than adequate for low power industry grade applications and this limit is where the optimum performance is preserved. If required, the performance can be extended to higher frequencies by simply replacing the op-amp used.

Once the data acquisition node was ready for operation along with the required calibration setup, the autonomous 
calibration could begin. This could be realized in two ways: the user may either provide points remotely to the node or let the node use an intelligent mechanism to find appropriate points for the automatic calibration stage. This improved linearization method provides the means to model the input output transfer function to match the effects of the environment parameters depending on whether the user knows about the environment subject to monitoring. If the user may not know much about the environment, the device will intelligently perform the calibration. A reference sensor is also used to correctly map the inputs with the outputs, so that subsequently the transfer function of the sensor is obtained by the SRI algorithm after the required calibration points are derived and this can be done within the microprocessor or externally for much more computational accuracy if required. With the SRI algorithm running in the micro-processor, the accuracy of the acquired data has been evaluated with different sensors attached to the prototype sensor node. The test bench included a K-type thermocouple (AEM 30-2067), 10k NTC Thermistor (EPCOS B57867S0103 10k $\Omega @ 25^{\circ} \mathrm{C}$ ), Pt100 RTD (RS 611-8264) and a $20 \mathrm{~kg}$ Load Cell (VISHAY Model 9010 - 2mV/V). Initially all sensors were tested with both voltage and current excitation supplies for best accuracy. The results were compared with the improved linearization method output to determine the effectiveness of proposed method. Table IV shows the comparison result.

TABLE IV

ACCURACY COMPARISON WITH SRI METHOD

\begin{tabular}{|l|l|l|}
\hline \multirow{2}{*}{ Sensor } & \multicolumn{2}{|c|}{ Best Accuracy } \\
\cline { 2 - 3 } & Without SRI & With SRI \\
\hline $\begin{array}{l}\text { K type } \\
\text { Thermocouple }\end{array}$ & $\pm 3 \mathrm{C}^{\circ}$ & $\pm 0.8 \mathrm{C}^{\circ}$ \\
\hline Thermistor & $\pm 1 \mathrm{C}^{\circ}$ & $\pm 0.5 \mathrm{C}^{\circ}$ \\
\hline Pt100 RTD & $\pm 3 \mathrm{C}^{\circ}$ & $\pm 0.4 \mathrm{C}^{\circ}$ \\
\hline Load Cell & $\begin{array}{l} \pm 250 \mathrm{~g}(\approx 1.3 \% \text { full } \\
\text { scale })\end{array}$ & $<0.5 \%$ full scale \\
\hline
\end{tabular}

Here the proposed SRI algorithm can be successfully used in micro processor software to compensate signal errors present at the measurement. Further, the fast execution time of the algorithm provides a feasible time schedule for WSN event management. The prototype implementation of the WSN node is shown in Fig 11.

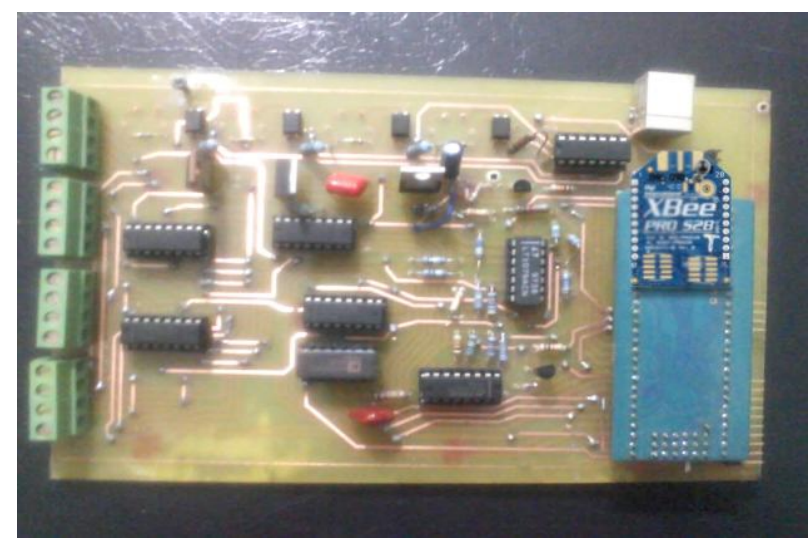

Fig. 11. Prototype Implementation

\section{CONCLUSIONS AND FUTURE EXPLORATION}

The proposed UTIM concept has a highly flexible front end design for wireless sensor node technologies which reconfigures its hardware in an autonomic way to interface with a wide range of distributed sensors to solve the multisensing challenge encountered in many WSN applications where different types of sensors are to be supported. Proposed Simplified Rational Polynomial Interpolation technique provides a robust linearization for sensors in order to maintain least amount of execution time required for reconfigurable hardware systems. Power consumption tests prove that UTIM is highly power efficient and can be integrated with an ultra low power processing unit (e.g. Nano Watt) in a WSN node. The circuit can be further miniaturized through surface mount devices. For future work, the development of adaptive reconfigurable hardware designs, autonomous sensor identification and sensor plug and play technologies will be evaluated to introduce a better deployment for distributed WSN applications. New possible algorithms that follow in the path of rational interpolation [35] will be explored to improve sensor linearization. Design improvements and miniaturization through newer high end versions of Programmable System-On-Chip integrated mixed signal devices will be considered with the required reconfigurability and the capable computational power. Other signal processing techniques will be also integrated to optimize the final solution.

\section{ACKNOWLEDGMENT}

The authors would like to thank the Managing Director of Attotech System Engineering (PVT) Ltd and senior staff of Sri Lanka Institute of Information Technology, Malabe for providing hardware accessories, laboratory facilities to accomplish this task.

\section{REFERENCES}

[1] J. T. John and S. R. J. Ramson, "Energy-Aware Duty Cycle Scheduling for Efficient Data Collection in Wireless Sensor Networks", IJARCET Volume 2, Issue 2, Feb . 2013.

[2] Shenfang Yuan, Lei Qiu, Shang Gao, "Providing Self-Healing Ability for Wireless Sensor Node by Using Reconfigurable Hardware", MDPI Sensor Journal, Oct. 2012.

[3] K. M. Iswarya, S. Usha, "Adaptive Runtime Reconfigurable Wireless Sensor Nodes Based On Dynamical Data Aggregation", IJREAT Volume 1, Issue 1, March. 2013.

[4] Rentao Wang, Ken Deevy, "Reconfigurable Adaptive Wireless Sensor Node", ISSC 2012, NUI Maynooth, June .28, 2012.

[5] N.I. Khachab, M. Ismail, "Linearization Techniques for n-th Order Sensor Model in Mos VLSI Technology". IEEE Trans. Circuits Syst. 1991, 38, 1439-1449.

[6] Z. Guangbin, S. Sooping, L. Jin, S. Sterrantino, D.K. Johnson, S. Jung, "An Accurate Current Source With On-Chip Self-Calibration Circuits for Low-Voltage Current-Mode Differential Drivers". IEEE Trans. Circuits Syst., 2006, 53, 40-47.

[7] G.E. Iglesias, E.A. Iglesias, "Linearization of Transducer Signal Using an Analog to Digital Converter". IEEE Trans. Instrum. Meas. 1988, 37, 53-57.

[8] B. Vargha, I. Zoltán, "Calibration Algorithm for Current-Output R-2R Ladders". IEEE Trans. Instrum. Meas. 2001, 50, 1216-1220.

[9] P. Malcovati, C.M. Leme, P. O'Leary, F. Maloberti, H. Baltes, "Smart sensor interface with A/D conversion and programmable calibration". IEEE J. Solid-State Circuits 1994, 29, 963-966.

[10] L. E. Bengtsson, "Lookup Table Optimization for Sensor Linearization in Small Embedded Systems", Journal Sensor Technology, doi:10.4236/jst.2012.24025, Dec.2012.

[11] Anuradha C. Ranasinghe, Lahiru K. Rasnayake, M. Kalyanapala, "Reconfigurable Universal Sensor Interface for Distributed Wireless Sensor Node". IEEE ICTER. 2013, Colombo. 
[12] Anuradha C. Ranasinghe, Lahiru K. Rasnayake, M. Kalyanapala, "Development of Intelligent Reconfigurable NCAP for Smart Transducer Applications". IEEE In Proc, CARE2013, Jabalpur, India 2013.

[13] R.A. Hooshmand, M. Joorabian, "Design and optimization of electromagnetic flow meter for conductive liquids and its calibration based on neural networks". IEE Proc. Sci. Meas. Technol. 2006, 153, 139-146.

[14] J.C. Patra, A.E. Luang, P.K. Meher, "Novel Neural Network-based Linearization and Auto compensation Technique for Sensors". Presented at the IEEE International Symposium on Circuits and Systems, Island of Kos, Greece, May 2006. Sensors 2008, 87427

[15] J. Tao, P. Qingle, L. Xinyun, "An Intelligent Pressure Sensor Using Rough Set Neural Networks". In Proceedings of the IEEE International Conference on Information Acquisition, Shandong, China, July 2006.

[16] C.A. Zoric, D. Martinovic, S. Obradovic, "A Simple 2D Digital Calibration routine for Transducers". FACTA UNIVERSITATIS (NIS ) SER.: ELEC. ENERG. vol. 19, no. 2, August 2006, 197-207.

[17] W.T. Bolk, "General Digital Linearising Method for Transducers". J. Phys. E: Sci. Instrum., Vol. 18, 1985. Great Britain.

[18] L.K. Fouad, G. Horn, J.H. Huijsing, "A Noniterative Polynomial 2-D Calibration Method Implemented in a Microcontroller". IEEE Trans. Instrum. Meas. 1997, 46, 752-757.

[19] J. Rivera, M. Carrillo, M. Chacon, G. Herrera, G. Bojorquez, "Improved Progressive Polynomial Algorithm for Self-Adjustment and Optimal Response in Intelligent Sensors". Sensors (ISSN 14248220; CODEN: SENSC9). 2008, 8, 7410-7427.

[20] Y. Hu, V. Tao, "A Comprehensive Study of the Rational Function Model for Photogrammetric Processing", Photogrammetric Engineering \& Remote Sensing, Vol. 67, No. 12, December 2001, pp. 1347-1357.

[21] Yuan Tian, Eylem Ekici, "Energy-Constrained Task Mapping and Schedualling in Wireless Sensor Networks", IEEE International Conference on Mobile Adhoc and Sensor Systems Conference, Nov. 7 2005.

[22] J. Stoer, R. Bulirsch, "Introduction to Numerical Analysis", Second Edition, Springer-Verlag New York, Inc. 1980, 1993.

[23] K.E. Atkinson, "An Introduction to Numerical Analysis", Second Edition, John Wiley \& Sons Inc, US 1989.

[24] J. Fraden, "Handbook of Modern Sensors-Physics, Designs and Applications", Fourth Edition, Springer, ISBN 978-1-4419-6465-6

[25] A. Pasic, J. Dowling, "Linearising Calibration Methods for a Generic Embedded Sensor Interface (GESI)", 1st International Conference on Sensing Technology, November 21-23, 2005 Palmerston North, New Zealand

[26] H. Yamasaki, "Intelligent Sensors", Yokogawa Research Institute Corporation Tokyo, Japan

[27] G. Walberg, "Cubic spline Interpolation: A Review", Department of Computer Science, Columbia University, New York, NY 10027

[28] J. Bartkovjak, M. Karovičová, "Approximation by Rational Functions", Institute of Measurement Science, Slovak Academy of Sciences Dúbravská cesta 9, 84219 Bratislava, Slovakia.

[29] C.T. Kelley, "Iterative Methods For Optimization", Society For Industrial and Applied Mathematics, 1999

[30] L. Burden, J. Faires, "Numerical Analysis", $7^{\text {th }}$ Edition.

[31] N. Carlson, "NURBS Surface Fitting with Gauss-Newton", Institute of Measurement Science, Slovak Academy of Sciences Dúbravská cesta 9,842 19 Bratislava, Slovakia

[32] C. L Karr, D. A. Stanley, and B. J. Scheiner, "Genetic Algorithm Applied to Least Squares Curve Fitting"

[33] Wilfiam K. George, Paul D. Beuther, Aamir Shabbir, "Calibrations for Hot Wires in Thermally Varying Flows", Turbulence Research Laboratory, Department of Mechanical and Aerospace Engineering, University at Buffalo, SUNY.

[34] NIST/SEMATECH, "e-Handbook of Statistical Methods", http://www.itl.nist.gov/div898/handbook/, 2012

[35] Chang Wen Li, Xiao Lin Zhu, Le Zou, "Modified Thiele-Werner Rational Interpolation", Journal of Mathematical Research \& Exposition, Jul 2010 